\title{
Die Zürcher Landschaftsschule
}

\section{Die ältere und die jüngere Zürcher Schule}

HANS BOESCH sprach im Jahre 1945 in seinem Nachruf auf seinen Vorgänger HANS J.WEHRLI von der «Zürcher Schule» der Landschaftskunde (Verh.d. Schweiz. Naturf. Ges.). Diese zeichne sich dadurch aus, daß sie die «Synthese in der Landschaftskunde» betone. Gemeint war «die Verbindung von naturmit geisteswissenschaftlichen, vor allem historischen Arbeitsmethoden». Im Unterschied zur naturwissenschaftlich orientierten Geographie in Deutschland um die Jahrhundertwende führe die «kulturlandschaftsgeschichtliche Betrachtung» der Zürcher Schule weit darüber hinaus. Daß sich der damals 34 Jahre junge Direktor des Geographischen Instituts zu profilieren suchte, indem er sich von der durch die Geopolitik korrumpierten reichsdeutschen Geographie distanzierte, ist auf dem zeitgenössischen Hintergrund durchaus verständlich, wenn auch die Akzente reichlich undifferenziert gesetzt wurden. Wenn heute von der Zürcher Landschaftsschule gesprochen wird, ist jedoch nicht diese ältere Gruppe anvisiert (WEHRLI, LEUTENEGGER, EGLI, WINKLER, BOESCH), sondern die von mir als «jüngere Schule» bezeichnete Gruppe um CAROL und WERNLI, die sich vielfach auf HANS BOESCH als ihren Mentor beruft (oder ist es nicht mehr als Reverenz?)

Daß WINKLER und CAROL die beiden Hauptexponenten der beiden Zürcher Landschaftsschulen sind, braucht wohl nicht näher erläutert zu werden; in ihren methodologisch-programmatischen Aufsätzen wirkten sie weit über die Landesgrenzen hinaus. Ihre innere Verwandtschaft ist denn auch größer als ihr äußeres Gehabe. Es spricht m. E. viel dafür, daß das CAROLsche «Sphärenmodell» auf Überlegungen zurückgeht, welche WINKLER schon in den 30er Jahren anstellte, namentlich, wenn er vom «spezifischen Gegenstand» der Geographie spricht, als dem «... in den (geographischen) Landschaften und der Erdhülle als Landschaftskomplex zum Ausdruck kommende(n) Gesamteffekt der Korrelation Lithosphäre-Hydrosphäre-Atmosphäre-Biosphäre» wINKLER 1946/339f).

Dr. Hans R. Brunner, Sonnenhaldenstraße 10B. 9008 St. Gallen.

\section{Die "Landschaft» eine Erfindung des 19. Jahrhunderts}

Jahrhundertelang war die Geographie (oder Erdkunde) die Beschreibung (Deskription) von Land und Leuten. Der besseren Operabilität wegen wurden überschaubare Beschreibungseinheiten gebildet und mit "geographischen» Namen versehen. Die Abgrenzungen wurden mehr oder weniger nach Gutdünken gezogen. Offenbar «...in dem Bestreben, den geographischen Betrachtungen Dauerwert zu verschaffen», wie BÜRGER (1935/7) schreibt, versuchte P. LEYSER um 1726 "natürliche» geographische Einheiten zu kreieren. Dabei entbrannte ein Streit darüber, ob den «trockenen» Grenzen (Berge) oder den «nassen» (Flüsse) der Vorzug zu geben sei. Einen Schritt weiter gingen ZEUNE und BUCHER, zwei Vertreter von trockenen Grenzen. Sie postulierten die Wasserscheiden als «natürliche Pflanzen-, Tierund Völkerscheiden». Es handelt sich demnach um Landschaften «mit einer besonderen inneren Gesetzlichkeit». Nach BURGER (1935/12) erhielt die geographische Landschaft zum ersten Mal «den Charakter einer höheren Einheit». Während HUMBOLDT noch von der «Einsicht in den inneren $\mathrm{Zu}$ sammenhang der Naturkräfte» und «in das harmonische Zusammenwirken der Kräfte» spricht, ist für CARL RITTER die Landschaft ein individueller Organismus, der seine hervorragende Bedeutung durch den Menschen erhält. Wenn auch RITTER mehr an die Großlandschaften (die Erdteile) dachte, so konnten sich die späteren Landschaftsgeographen doch auf diese durch und durch romantische Idee von der harmonischen Verschmelzung von Natur und Geist bei ihm berufen. Die RITTERsche Ansicht, wonach die Erde - als umfassender Organismus - nicht nur die Wohn- und Wirkungsstätte, sondern vielmehr noch «das Erziehungshaus, die große Erziehungsanstalt des Menschengeschlechts» sei, pervertierte gegen Ende des Jahrhunderts nicht ohne Schuld RATZELS zum platten Determinismus, wonach das $\mathrm{Na}$ turmilieu (v. a. das Klima) den Charakter des Menschen und seiner Werke (auch geistige wie den Staat) kausal bestimme. Bei RATZEL ist «die geographische Auffassung der Dinge» das "Erfassen des Landschaftlichen, d.h. des Nebeneinander- und Miteinandervorkommens in der Natur» schlechthin 
(1904/12). Landschaftskunde kann danach «nicht rein auf dem wissenschaftlichen Boden gedeihen..., denn von ihr fordert man Bilder, so muß sie von der dichterischen Wiedergabe der Natureindrücke und von der Landschaftsmalerei lernen, wie man die geographischen Erscheinungen einer Erdstelle oder eines größeren Gebietes zu geschlossenen Bildern vereinigt» (S. 14). Landschaftskunde ist bei RATZEL ein «Zweig der beschreibenden Geographie» (S. 14), also etwas zwischen Kunst und Wissenschaft, ohne beides ganz zu sein. Weder bei RATZEL (1844-1904 noch bei HETTNER (1859-1941), dem anderen Geographiepapst, war die Geographie reine Landschaftskunde. Beide betrachteten die Geographie als eine chorologische Wissenschaft, d. h. eine Wissenschaft, welche beliebige Dinge unter einen räumlichen Aspekt ordnet. Landschaftliche Schilderung ist gewissermaßen eine expressionistische Ergänzung der Geographie. Die Epigonen RATZELS und HETTNERS setzten sich aber mehr und mehr über die subtile Grenze zwischen Kunst und Wissenschaft hinweg, mangels Kunstverstandes. Behaviorismus und Gestaltpsychologie feierten Urstände und so kamen einige Geographen auf den gloriosen Einfall, die "Landschaft» zum "Hauptbegriff» und «Inbegriff» der Geographie zu stilisieren. Unter einer «geographischen» Landschaft hatten Geographen inskünftig zu verstehen «...einen Teil der Erdoberfläche, der nach seinem äußeren Bilde und dem Zusammenwirken seiner (Erscheinungen) sowie den inneren und äußeren Lagebeziehungen eine Raumeinheit von bestimmtem Charakter bildet, der diesen Erdraum von seiner Umgebung unterscheidet. In der lebensvollen und möglichst naturwahren Darstellung solcher landschaftlicher Einheiten gipfelt alle geographische Arbeit» so BÜRGER (1935/29). Neu war nicht die Idee, sondern die einseitige Überziehung des Gedankens.

\section{Die legitimatorische Funktion}

Die expressionistische Wende in der Geographie (der deutschen) um 1920 kann nicht allein aus der damaligen Kunstszene heraus begriffen werden. Dazu war die Affinität zur Kunst zu oberflächlich. Vielmehr müssen damit die politischen Vorgänge verbunden werden. Ähnlich wie früher zu Napleons Zeiten und später nach 1945 können deutsche Geographen plötzlich nichts mehr anfangen mit Staatsgrenzen. Immer dann, wenn deutsche Grenzen ins Wanken geraten, d. h. wenn sie enger werden, wird der Staatsbegriff in der Geographie suspekt. Immer dann wird die «reine» Geographie auf den Schild gehoben. Politische Geographie oder Länderkunde ist nur in Expansionsphasen gefragt. Die politischen Absichten dieser Entpolitisierung der Geographie werden regelmäßig mit der vorgeschobenen Wissen- schaftlichkeit des Faches verschleiert. So tönt es bei BÜRGER: «Innerlich selbständig ist die Geographie nur, wenn sie einen eigenen Erdraumbegriff besitzt... Deshalb mußte die Geographie, wollte sie den Rang einer selbständigen Wissenschaft für sich in Anspruch nehmen, loskommen von dem an sich ungeographischen Begriff des Staates als ausschließlicher Grundlage geographischer Erdraumerfassung und sich einer Erdraumeinheit zuwenden, die für sie spezifisch und deren Erfassung und Darstellung wissenschaftlich notwendig ist» (1935/27f).

Die Landschaft ist für die Geographen der Zwischenkriegszeit eine «objektiv gegebene und natürliche Einheit» und daher alleiniges und ausschließendes Objekt der Geographie, kurz ihr «Inbegriff» (WINKLER 1951/137ff). Eine erkenntnislogische Hinterfragung erfolgt immer nur von den Gegnern. Die Verfechter der Landschaftsidee argumentieren mit Vorliebe etymologisch und polemisch: Wer die "Landschaft» nicht als zentrale Denkfigur akzeptiert, ist gegen das Fach Geographie überhaupt. EMIL EGLI findet es (1967) erstaunlich, daß es in der Geographie eine Diskussion gibt, welche nach dem Gegenstand fragt. Das könne doch nur die Gefahr einer Verwirrung heraufbeschwören. Mit einer Zähigkeit sondergleichen versuchen sich die Landschaftsgeographen einzureden, nur mit einem anerkannten Objekt könne man Wissenschaft treiben, und dieses Objekt könne für die Geographie nichts anderes sein als eben die Landschaft.

\section{Von der Ganzheitlichkeit zur Totalität - oder: «Die Selbstauflösung der Landschaftsgeographie»}

Während WINKLER und EGLI weiterhin an der Ganzheitlichkeit der Landschaft festhielten (vgl. EGLI 1967/3), kam m. E. bei HANS BOESCH immer mehr eine gewisse Zurückhaltung gegenüber dem Landschaftsbegriff zum Tragen. Methodologisch schlug sich dies aber erst bei seinen Schülern HANS CAROL und OTTO WERNLI nieder. Beide wenden sich vehement gegen die Ganzheitsauffassung der Landschaft. Dabei handelt es sich nach ihrer Meinung um «bloße Behauptungen» und nicht um «bewiesene Tatsachen». Im Vordergrund steht wieder einmal das Problem der Abgrenzungen von Landschaften. Nach CAROL und WERNLI steht und fällt die Ganzheitlichkeit mit objektiven und auch allgemein anerkannten Abgrenzungen, welche ein Landschaftsindividuum von anderen klar scheiden könnte. Da solche objektiven Abgrenzungen of fenbar nicht existieren, ist für sie auch die Ganzheitshypothese nicht haltbar. Den jüngeren Landschaftsgeographen geht es vorwiegend darum, Landschaften beliebig zu begrenzen und zu gruppieren, je nach Zweck und Interesse der Untersuchung. Sie wollen es zwar nicht 
wahrhaben, daß dadurch «Landschaft» oder «Geomer» zu einer austauschbaren Chiffre für das Untersuchungsgebiet wird. Landschaft wird dadurch zum Bezugsobjekt. Es bleibt schleierhaft, warum die Landschaft weiterhin Forschungsobjekt der Geographie sein soll, wenn man es völlig nach freiem Ermessen definieren darf. Was heißt denn schon: «Die Landschaft ist also ein spezifisches Korrelationsgefüge der sie aufbauenden Sphären» (CAROL 1956/116). Das sind doch alles menschliche Konstrukte und weit davon entfernt, «konkrete Objekte» zu sein. Bei Sätzen wie: «Unter dem Begriff Landschaft verstehen wir etwas durchaus Konkretes: die ungeheure Fülle irdischen Daseins» (CAROL $1956 / 114$ ) habe ich die starke Vermutung, daß es sich um Tautologien handelt. Wenn «konkret» nicht etwas anderes heißen soll als "anschaulich, greifbar» und wohl auch «be-greifbar», so ist eine Fülle irdischen Daseins deshalb «ungeheuer», weil sie nicht anschaulich genannt werden kann. Aber offenbar muß die Umschreibung von Landschaft derart «totalitär» sein, daß sie so ziemlich alles umfassen kann. Von der «astronomischen» Geographie über die "quantitative» bis hin zur individuellen Freizeitverhaltensgeographie und neuerdings zur «timegeography» sollte alles unter einen Hut gebracht werden können. Geographie als Totalwissenschaft, als "Soziologie aller Soziologien», wie ERNST WINKLER einmal allen Ernstes meinte, da die Landschaft als Objekt der Geographie «...ja selbst eine Sozietät» sei und zwar nicht nur irgend eine Sozietät, sondern als "Korrelat aller terrestrischen Seinssphären..., die Sozietät der Sozietäten» (WINKLER 1951/53). Die Geographie als Landschaftskunde ist es daher allein (nach WINKLER), welche «die vollkonkrete, nämlich landschaftsverhaftete Gemeinschaft der Menschen in ihrer landschaftlichen Gliedhaftigkeit zu erkennen trachtet» (WINKLER 1964). Daß sie es ganz offenbar nicht vermag, liegt nicht nur an solchen "sophisticated» Formulierungen schwer nachvollziehbarer Vorstellungen, das zeigen auch die praktischen Ergebnisse der geographischen Landschaftsforschung, sofern sie an der ganzheitlichen und totalitären Konzeption festhalten. EMIL EGLI geht diesen Schwierigkeiten aus dem Wege durch eine literarische Erhöhung seines landschaftlichen Anliegens, was ihm erlaubt, ein breites außerfachliches Publikum anzusprechen.

Im Gegensatz zur älteren Zürcher Schule verliert bei CAROL und WERNLI der Landschafts- und Geomer- begriff seinen Objektcharakter weitgehend durch die geosphärische Konstruktion auf dem Korrelatbegriff. Landschaft steht für das Ziel geographischen Forschens nach dem Zusammenwirken, resp. den Wechselwirkungen der verschiedenen Geosphären. Offenbar unbemerkt aber wird das schon vorausgesetzt, wonach man forschen möchte, das man beweisen müßte. Geographie als Wissenschaft sollte strikte sich beschränken auf das methodisch saubere Auf- und Nachweisen räumlich-kausaler Beziehungen zwischen Variablen menschlicher Daseinsformen.

Erlauben Sie mir mit einem Zitat des Geographiehistorikers HANNO BECK zu schließen:

«Es ist unnötig, Studenten und jeden an der Geographie Interessierten mit der Scheinlogik des Landschaftsbegriffes $\mathrm{zu}$ ängstigen und $\mathrm{zu}$ verwirren. Landschaft ist eine mehr oder weniger große, sich dem menschlichen Geist notwendigerweise darbietende kleinere Einheit des Beschreibens und Erklärens in der Geographie; sie darf subjektiv, aber möglichst zweckmäßig, gebildet, muß aber objektiv erforscht werden. Geographie kann auch ohne den Begriff Landschaft auskommen, der nie eine allgemeingültige extensio besitzt» (BECK 1973/328).

\section{Literatur}

BECK, HANNO. 1973. Geographie. Freiburg und München. BURGER, KURT. 1935. Der Landschaftsbegriff. Dresden.

CAROL, HANS. 1956. Zur Diskussion um Landschaft und Geographie. In: Geogr. Helv. IX, S. 111-133.

CAROL, HANS. 1963. Zur Theorie der Geographie. In: Mitt. der Österr. Geogr. Gesellschaft, Bd. 105, Heft I/II, S. 23-38.

EGLI, EMIL. 1967. Landschaft und Geographie. In: Mitt. der Naturforschenden Ges. Schaffhausen, Bd. XXVIII, Jg. 1963/67, S. 1-14.

LEUTENEGGER, ALBERT. 1922. Begriff, Stellung und Einteilung der Geographie. Gotha.

RATZEL, FRIEDRICH. 1904 (1923, 4. Aufl.). Úber Naturschilderung. München und Berlin.

SCHULTZ, HANS-DIETRICH. 1980. Die deutschsprachige Geographie von 1800 bis 1970 . FU Berlin, Abh.d. Geogr. Instituts Anthropogeographie Band 29.

WERNLI, OTTO. 1958. Die neuere Entwicklung des Landschaftsbegriffes. Diss. Uni Zürich.

WINKLER, ERNST. 1946. Das System der Geographie und die Dezimalklassifikation. In: Geogr. Helv. I, S. 337-349.

WINKLER, ERNST. 1951. Landschaft als Inbegriff der Geographie. In: Geogr. Helv. VI, S. 137-140.

WINKLER, ERNST. 1977. Der Geograph und die Landschaft. Festschrift zum 70. Geburtstag. Atlantis Zürich. 